\title{
A DRIFT ESTIMATOR FOR NON-LINEAR STOCHASTIC PROCESSES
}

\author{
Erik Honobe* \\ Departamento de Finanzas, Tecnológico de Monterrey, \\ Campus Estado de México \\ Jonathan Sampson \\ Departamento de Ingeniería Industrial, Tecnológico de Monterrey, \\ Campus Estado de México
}

(Received 3 March 2003, accepted 18 May 2003)

\begin{abstract}
In this paper, we propose a drift estimation method for non-linear stochastic processes. In particular, we focus on producing strongly consistent estimators in the case of discrete observations, and present a proof of convergence of these estimators. Potential applications of these estimators include detecting cyclical and other non-linear trends inherent to asset prices.
\end{abstract}

\section{Resumen}

En este trabajo se presenta un método de estimación para procesos estocásticos no lineales. En particular nos interesa obtener estimadores consistentes en el caso de datos discretos y una prueba de convergencia para éstos. Las aplicaciones de dichos estimadores permite observar si existen tendencias ciclícas y no lineales inherentes a los precios de los activos.

JEL classification: $C 13, C 60$

Keywords: Estimation, Mathematical Methods

* Departamento de Finanzas, Tecnológico de Monterrey, Campus Estado de México. Carretera Lago de Guadalupe Km. 3.5, Col. Margarita Maza de Juárez, Atizapán de Zaragoza, 52926, Estado de México. Telephone +52(55)58645636. E-mail: ehonobe@itesm.mx

The authors are grateful to the anonymous referees for their comments. 


\section{Introduction}

Stochastic processes are widely used in financial modeling, since they have numerous applications in many areas of finance. Further, while estimates of the risk and return of assets will directly affect, say, investment decisions of portfolio managers, the assumptions involved in deriving these estimates are as important as the estimates themselves. Moreover, their validity is crucial to quality investment decision-making.

In particular, the assumption that asset prices are linear stochastic processes is prevalent in modeling stock returns, for its immediate applications and strongly consistent parameter estimations (Zane (1994), Nuñez-Mora (2002)). There is also a growing interest for non-linear models in mathematical finance. Indeed, non-linear models such as those of GARCH, dynamical chaos, cycles (periodic and aperiodic) are now used to attempt to explain various observed facts in the stock market, such as long memory in stock prices, heavy tails in the distributions of stock returns, cluster property of prices, and other phenomena which cannot be understood in the framework of linear models (Shiryaev (1999)).

In this paper, we propose a drift estimation method for non-linear stochastic processes whose non-linear elements are known functions. While this last assumption might seem somewhat restrictive, we note that it can readily be relaxed to merely assuming that the non-linear elements belong to a given set of non-linear functions, and that they could potentially be determined through some computerized trial-error process involving the estimation method presented here.

This paper is organized as follows. Section 2 defines the non-linear stochastic processes that are to be estimated. Section 3 introduces the estimation method of drift coefficients when observations are continuous. The main contribution of this paper is then presented in Section 4, where we propose a drift estimation method for observations that are a discrete sampling of a non-linear stochastic process. In this section, we provide a formal proof that the proposed discrete drift estimator converges to its continuous counterpart as the length of the time intervals tends to zero. Section 5 focuses on the upward bias in the discrete estimators and applies recursive equations from Zane (1994) to produce unbiased estimators. Finally, a simulation and its results are shown in Section 6.

\section{Non-linear Stochastic Processes}

We start by defining the non-linear stochastic processes to estimate. Let

$d=$ number of stocks in the portfolio,

$d^{\prime}=$ number of possible economic states,

$P_{j}=$ probability of economic state $j$ to occur $\left(j=1, \ldots, d^{\prime}\right)$,

$S_{i j}=i$-th stock return for one time period $(i=1, \ldots, d)$ under economic state $j$

$$
\mathrm{E}\left(S_{i}\right)=\sum_{j=1}^{d^{\prime}} P_{j} S_{i j}
$$




$$
\sigma=\left(\sigma_{i j}\right)_{i, j=1}^{d, d^{\prime}}, \sigma_{i j}=\sqrt{P_{j}}\left[S_{i j}-\mathrm{E}\left(S_{i}\right)\right]
$$

Then $A=\sigma \sigma^{T}$ is the covariance matrix for the portfolio. Further, the bilinear stochastic differential equations

$$
\mathrm{d} X_{i}(t)=b_{i} X_{i}(t) \mathrm{d} t+\sum_{j=1}^{d^{\prime}} \sigma_{i j} X_{i}(t) \mathrm{d} W_{j}(t),
$$

$i=1, \ldots, d, X_{i}(0)=x_{0} ;$ some $x_{0}$, can be used for modeling the stock returns. Indeed, they represent a $d$-dimensional Itô process, because $W$ is a $d^{\prime}$-dimensional Brownian motion and $v_{i j}=\sigma_{i j} X_{i}(t)$ is a $\mathcal{B} \times \mathcal{F}$-measurable, $\mathcal{F}_{t}$-adapted function such as the functions usually defined for Itô processes (Øksendal (1998)).

In this paper, we propose an estimation method for stock prices following the non-linear Itô process:

$$
\mathrm{d} X_{i}(t)=\left[b_{i}+\Psi_{i}(t)\right] X_{i}(t) \mathrm{d} t+\sum_{j=1}^{d^{\prime}} \sigma_{i j} X_{i}(t) \mathrm{d} W_{j}(t),
$$

where $\Psi_{i}$ is a known function of $t$ such that $\int_{0}^{t} \Psi_{i}(s) \mathrm{d} s$ is a continuous function of $t$. The definition of $\Psi_{i}$ as a function of time is consistent with the hypothesis that some assets prices exhibit trends of accelerating growth and/or decay, slowing growth and/or decay, cyclical trends and other trends that solely depend on time.

\section{Drift Estimators in the Continuous Case}

We examine the case of continuous observations and the corresponding drift estimators. It has been shown (Zane (1994)) that, for the continuous case of equations such as $(2.0)$,

$$
\bar{b}_{i}^{\prime}(t)=\frac{1}{t} \int_{0}^{t} \frac{\mathrm{d} X_{i}(s)}{X_{i}(s)}
$$

is a strongly consistent estimator, i.e.

$$
\lim _{t \rightarrow \infty} \bar{b}_{i}^{\prime}(t)=b_{i}
$$

Hence, for the Itô process in (2.1), we let

$$
\bar{b}_{i}(t)=\frac{1}{t}\left[\int_{0}^{t} \frac{\mathrm{d} X_{i}(s)}{X_{i}(s)}-\int_{0}^{t} \Psi_{i}(s) \mathrm{d} s\right]
$$

and, from (2.1), we obtain 


$$
\begin{aligned}
\bar{b}_{i}(t) & =\frac{1}{t}\left[\int_{0}^{t}\left[b_{i}+\Psi_{i}(s)\right] \mathrm{d} s-\sum_{j=1}^{d^{\prime}} \sigma_{i j} \mathrm{~d} W_{j}(s)-\int_{0}^{t} \Psi_{i}(s) \mathrm{d} s\right] \\
& =b_{i}+\sum_{j=1}^{d^{\prime}} \sigma_{i j} \frac{W_{j}(t)}{t}
\end{aligned}
$$

and, as in (3.0), it follows that $\bar{b}_{i}(t)$ is a strongly consistent estimator, i.e.

$$
\lim _{t \rightarrow \infty} \bar{b}_{i}(t)=b_{i}
$$

\section{Drift Estimator for Discrete Observations}

We now focus on estimating drift coefficients when observations are a discrete sampling of a non-linear Itô process. We denote

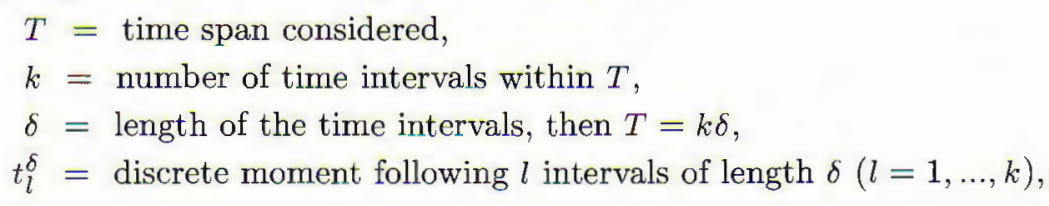

$$
\Phi_{i}^{\delta}(l)=\frac{X_{i}\left(t_{l}^{\delta}\right)-X_{i}\left(t_{l-1}^{\delta}\right)}{X_{i}\left(t_{l-1}^{\delta}\right)} .
$$

As proposed by Zane (1994), a discretization of the estimators in (3.0) is

$$
{\hat{b^{\prime}}}_{i}^{\delta}(k)=\frac{1}{k \delta} \sum_{l=1}^{k} \Phi_{i}^{\delta}(l) .
$$

We propose a discretization of the estimators in (3.1) for the non-linear Itô process in (2.1). Let

$$
\begin{aligned}
\varphi_{i}^{\delta}(l+1) & =\frac{\Phi_{i}^{\delta}(l+1)+1}{\exp \left\{\int_{t_{l}^{\delta}}^{t_{l+1}^{\delta}} \Psi_{i}(s) \mathrm{d} s\right\}}-1, \\
\hat{b}_{i}^{\delta}(k) & =\frac{1}{k \delta} \sum_{l=1}^{k} \varphi_{i}^{\delta}(l) .
\end{aligned}
$$

Proposition 1:

$$
\lim _{\delta \rightarrow 0} \hat{b}_{i}^{\delta}(k)=\bar{b}_{i}(t)
$$

Before proving Proposition 1, we start by considering the following three results. Result 1. Given $\varepsilon>0, \exists \delta^{\prime}>0$ such that $\exp \{\delta\}<1+\delta+\delta \varepsilon, \forall \delta \leq \delta^{\prime}$. 
Proof. From the basic properties of $\exp \{x\}$, we know that, given $\varepsilon>0, \exists \delta^{\prime}$ such that

$$
\left|\frac{\exp \{x+\delta\}-\exp \{x\}}{\delta}-\exp \{x\}\right|<\varepsilon, \quad \forall \delta \leq \delta^{\prime} .
$$

Thus, at $x=0$,

$$
\left|\frac{\exp \{\delta\}-1}{\delta}-1\right|<\varepsilon \Leftrightarrow 1+\delta<\exp \{\delta\}<1+\delta+\delta \varepsilon, \quad \forall \delta \leq \delta^{\prime}
$$

Result 2. Let $\Delta_{i}^{l}=\int_{t_{l}^{\delta}}^{t_{l+1}^{\delta}} F(s) \mathrm{d} s$, some $F$ such that $\int_{0}^{t} F(s) \mathrm{d} s$ is a continuous function of $t$. Then

$$
\lim _{\delta \rightarrow 0} \frac{1}{k \delta} \sum_{l=1}^{k} \exp \left\{\Delta_{i}^{l}\right\}=1+\frac{1}{T} \int_{0}^{T} F(s) \mathrm{d} s, \quad T=k \delta .
$$

Proof. For any $k, \delta>0$, we have

$$
\frac{1}{k \delta} \sum_{l=1}^{k}\left(1+\Delta_{i}^{l}\right)=1+\frac{1}{T} \int_{0}^{T} F(s) \mathrm{d} s .
$$

Now, fixing $i$, and by definition of $\Delta_{i}^{l}$, given $\delta^{\prime}>0, \exists \delta>0$ such that

$$
\Delta_{i}^{l}=\int_{t_{l}^{\delta}}^{t_{l+1}^{\delta}} \Psi_{i}(s)<\delta^{\prime}, \quad l=1, \ldots, k .
$$

Then, given $\varepsilon>0$ and applying Result $1, \exists \delta>0$ such that

$$
1+\Delta_{i}^{l}<\exp \left\{\Delta_{i}^{l}\right\}<1+\Delta_{i}^{l}+\delta \varepsilon, \quad l=1, \ldots, k,
$$

so that

$$
\frac{1}{k \delta} \sum_{l=1}^{k}\left(1+\Delta_{i}^{l}\right)<\frac{1}{k \delta} \sum_{l=1}^{k} \exp \left\{\Delta_{i}^{l}\right\}<\varepsilon+\frac{1}{k \delta} \sum_{l=1}^{k}\left(1+\Delta_{i}^{l}\right) .
$$

It follows that

$$
\lim _{\delta \rightarrow 0} \frac{1}{k \delta} \sum_{l=1}^{k} \exp \left\{\Delta_{i}^{l}\right\}=1+\frac{1}{T} \int_{0}^{T} F(s) \mathrm{d} s .
$$

Result 3. Let $\Phi_{i}^{\delta}(l)$ as defined in (4.0), then

$$
\log \left(\Phi_{i}^{\delta}(l+1)+1\right)=\int_{t_{i}^{\delta}}^{t_{i+1}^{\delta}} \frac{\mathrm{d} X_{i}(s)}{X_{i}(s)} .
$$


Proof. By definition, proving Result 3 is equivalent to proving

$$
\frac{X_{i}\left(t_{l+1}^{\delta}\right)}{X_{i}\left(t_{l}^{\delta}\right)}=\exp \left\{\int_{t_{l}^{\delta}}^{t_{l+1}^{\delta}} \frac{\mathrm{d} X_{i}(s)}{X_{i}(s)}\right\} \text {. }
$$

Without loss of generality, we assume $\delta<1$. Indeed, for $\delta \geq 1$, take $n \in \mathbb{N}$ such that $(\delta / n)<1$ and apply Result 3 for $(\delta / n)$ to obtain

$$
\prod_{n^{\prime}=1}^{n} \frac{X_{i}\left(t_{n^{\prime}+1}^{\delta / n}\right)}{X_{i}\left(t_{n^{\prime}}^{\delta / n}\right)}=\prod_{n^{\prime}=1}^{n} \exp \left\{\int_{t_{n^{\prime}}^{\delta / n}}^{t_{n^{\prime}+1}^{\delta / n}} \frac{\mathrm{d} X_{i}(s)}{X_{i}(s)}\right\}
$$

which is equivalent to Result 3 for $\delta$. First, let $\delta^{\prime}, k^{\prime}, t_{k^{\prime}}^{\prime}>0$ such that $\delta^{\prime} k^{\prime}=\delta$, $t_{0}^{\prime}=t_{l}$ y $t_{k^{\prime}}^{\prime}=t_{l+1}$. Then,

$$
\prod_{l^{\prime}=1}^{k^{\prime}}\left(\Phi_{i}^{\delta^{\prime}}\left(l^{\prime}\right)+1\right)=\frac{X_{i}\left(t_{l+1}^{\delta}\right)}{X_{i}\left(t_{l}^{\delta}\right)}
$$

Also, as in (4.2), given $\varepsilon_{1}>0, \exists \delta_{1}>0$ such that

$$
\begin{gathered}
1+\Phi_{i}^{1}\left(l^{\prime}\right)<\exp \left\{\Phi_{i}^{1}\left(l^{\prime}\right)\right\}<1+\Phi_{i}^{1}\left(l^{\prime}\right)+\delta_{1} \varepsilon_{1}, \\
l^{\prime}=1, \ldots, k_{1}, \quad \delta_{1} k_{1}=\delta, \quad \varepsilon_{1}<1, \quad \delta_{1}<1 .
\end{gathered}
$$

Now, let

$$
M=\exp \left\{\left(\sup _{t_{l}^{\delta} \leq t \leq t_{l+1}^{\delta}}\left\{X_{i}(t)\right\}-\inf _{t_{i}^{\delta} \leq t \leq t_{l+1}^{\delta}}\left\{X_{i}(t)\right\}\right) \delta\right\}
$$

Then,

$$
\prod_{l^{\prime}=1}^{k^{\prime \prime}}\left(\Phi_{i}^{\delta^{\prime}}\left(l^{\prime}\right)+1\right) \leq M, \quad \forall k^{\prime \prime} \leq k^{\prime}
$$

Thus, given $\varepsilon_{2}>0$, setting $\varepsilon_{1}=\frac{(1-\delta)}{M} \varepsilon_{2}$ and applying (4.3) and (4.4), we have

$$
\begin{aligned}
\prod_{l^{\prime}=1}^{k_{1}} \exp \left\{\Phi_{i}^{\delta_{1}}\left(l^{\prime}\right)\right\} & \leq \prod_{l^{\prime}=1}^{k_{1}}\left(1+\Phi_{i}^{\delta_{1}}\left(l^{\prime}\right)+\delta_{1} \varepsilon_{1}\right) \\
& \leq \prod_{l^{\prime}=1}^{k_{1}}\left(1+\Phi_{i}^{\delta_{1}}\left(l^{\prime}\right)\right)+M \sum_{l^{\prime}=1}^{k_{1}} \frac{k_{1} !}{\left(k_{1}-l^{\prime}\right) ! l^{\prime} !}\left(\delta_{1} \varepsilon_{1}\right)^{l^{\prime}} \\
& \leq \prod_{l^{\prime}=1}^{k_{1}}\left(1+\Phi_{i}^{\delta_{1}}\left(l^{\prime}\right)\right)+M \sum_{l^{\prime}=1}^{k_{1}}\left(k_{1} \delta_{1} \varepsilon_{1}\right)^{l^{\prime}} \\
& <\prod_{l^{\prime}=1}^{k_{1}}\left(1+\Phi_{i}^{\delta_{1}}\left(l^{\prime}\right)\right)+M \frac{1}{1-\delta} \varepsilon_{1} \\
& =\prod_{l^{\prime}=1}^{k_{1}}\left(1+\Phi_{i}^{\delta_{1}}\left(l^{\prime}\right)\right)+\varepsilon_{2} .
\end{aligned}
$$


Taking the limits as $\delta_{1} \rightarrow 0$, we obtain

$$
\exp \left\{\int_{t_{l}^{\delta}}^{t_{i+1}^{\delta}} \frac{\mathrm{d} X_{i}(s)}{X_{i}(s)}\right\}=\frac{X_{i}\left(t_{l+1}^{\delta}\right)}{X_{i}\left(t_{l}^{\delta}\right)} .
$$

Proof of Proposition 1. As defined (4.1),

$$
\hat{b}_{i}^{\delta}(k)=-1+\frac{1}{k \delta} \sum_{l=1}^{k} \exp \left\{\log \left(\Phi_{i}^{\delta}(l)+1\right)-\int_{t_{l-1}^{\delta}}^{t_{l}^{\delta}} \Psi_{i}(s) \mathrm{d} s\right\} .
$$

From Result 3, we obtain

$$
\hat{b}_{i}^{\delta}(k)=-1+\frac{1}{k \delta} \sum_{l=1}^{k} \exp \left\{\int_{t_{i-1}^{\delta}}^{t_{i}^{\delta}} \frac{\mathrm{d} X_{i}(s)}{X_{i}(s)}-\Psi_{i}(s) \mathrm{d} s\right\} .
$$

Finally, setting $F(s)=\frac{\mathrm{d} X_{i}(s)}{X_{i}(s)}-\Psi_{i}(s)$ and applying Result 2 completes the proof.

Remark: The fact that we could apply Result 2 to complete the proof is consistent with the usual assumption in mathematical finance that asset prices have no "jumps" in the continuous case (Karatzas and Shreve (1998)), so that $\int_{0}^{t} F(s)$ is a continuous function of $t$.

\section{Strongly Consistent Drift Estimators}

As Zane (1994) demonstrated, $\hat{b}_{i}^{\prime \delta}(k)$ is an upward-biased estimator, as it includes the continuous compounding of stock price movements, which is observed after any discrete time interval, whereas $b_{i}$ represents a mere nominal stock yield occurring in a continuous timeframe.

More precisely, it was shown by Zane that

$$
\lim _{k \rightarrow \infty} \hat{b}_{i}^{\prime \delta}(k)=\frac{\exp \left\{\delta b_{i}\right\}-1}{\delta} .
$$

To avoid overstating the expected returns as shown in (5.0), we use the strongly consistent estimation method proposed by Zane (1994). Let

$$
\begin{aligned}
& \hat{\bar{b}}_{i}^{\delta}(0)=\text { an arbitrary constant }, \quad i=1, \ldots, d . \\
& Q(\delta, i, k)=\delta \hat{b}_{i}^{\delta}(k)+1 .
\end{aligned}
$$

$\hat{\bar{b}}_{i}^{\delta}(k)$ is then recursively defined for every $i=1, \ldots, d$ as

$$
\hat{\bar{b}}_{i}^{\delta}(k)= \begin{cases}\frac{\log \{Q(\delta, i, k)\}}{\delta}, & \text { if } Q(\delta, i, k)>0 \\ \hat{\bar{b}}_{i}^{\delta}(k-1), & \text { otherwise. }\end{cases}
$$


It follows from Proposition 1 that the above recursive equations will correct the upward bias of the drift estimators for non-linear Itô processes.

\section{A Numerical Example}

We show here a numerical example of drift estimators for the non-linear Itô process defined in (2.1) with the parameters

$$
\begin{gathered}
\left(\begin{array}{l}
b_{1} \\
b_{2}
\end{array}\right)=\left(\begin{array}{l}
0.6 \\
0.5
\end{array}\right), \\
\left(\begin{array}{ll}
\sigma_{11} & \sigma_{12} \\
\sigma_{21} & \sigma_{22}
\end{array}\right)=\left(\begin{array}{ll}
0.7 & 0.7 \\
1.0 & 0.5
\end{array}\right), \\
\left(\begin{array}{ll}
A_{11} & A_{12} \\
A_{21} & A_{22}
\end{array}\right)=\left(\begin{array}{ll}
0.98 & 1.05 \\
1.05 & 1.25
\end{array}\right), \\
\Psi_{1}(t)=\frac{\sin \left(\frac{t}{30}\right)}{100}, \quad \Psi_{2}(t)=\frac{\cos \left(\frac{t}{30}\right)}{100} .
\end{gathered}
$$

Using the recursive equations (5.1) on a simulation with a time span $T=2500$ and a time step $\delta=0.1$, we obtain the drift estimations

$$
\left(\begin{array}{l}
\hat{\bar{b}}_{1} \\
\hat{\bar{b}}_{2}
\end{array}\right)=\left(\begin{array}{l}
0.5986 \\
0.4938
\end{array}\right),
$$

which are shown in Graph 1 and are consistent with the results of Proposition 1 .

Graph 1. Drift Esstimators for Non-linear Itô Processes.

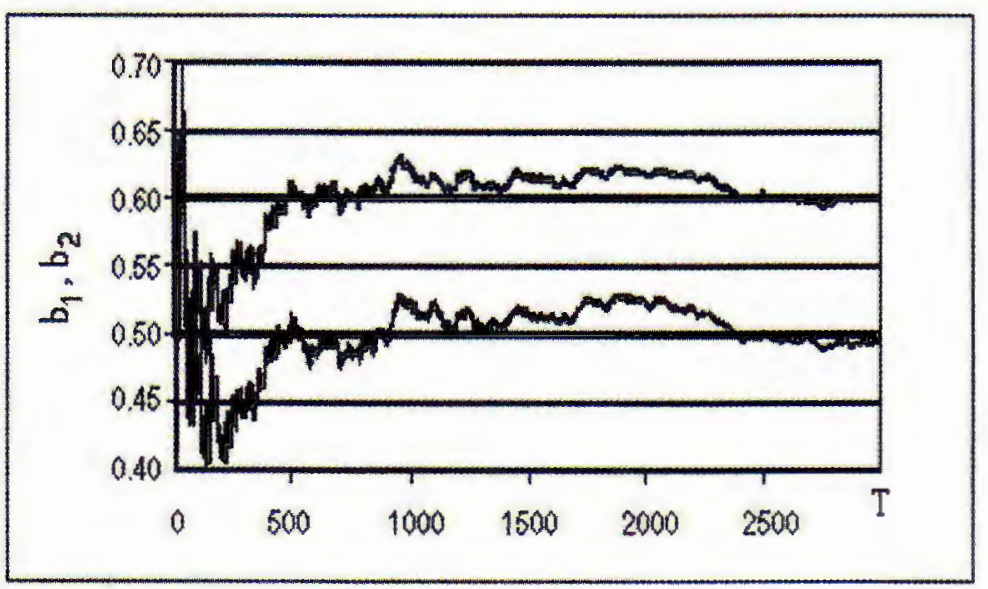




\section{Conclusion}

We have presented a drift estimator for non-linear Itô processes, as well as a proof that its discretization converges as expected when the length of the time intervals approaches zero. Further, while not shown in this paper, it can be inferred that this discretization also follows the other convergence properties of estimators for bilinear stochastic differential equations established by Zane (1994), and that the corresponding diffusion coefficients can also be estimated in a similar fashion. Therefore, if non-linear elements can be effectively identified through the use of some computerized trial-and-error procedure, the strongly consistent estimators proposed by Zane (1994) for linear stochastic processes could be generalized to a wide range of non-linear cases through the estimation method presented in this paper.

\section{References}

Karatzas, I. and S. E. Shreve (1998). Methods of Mathematical Finance. Springer-Verlag, New York.

Nuñez-Mora, J. A. (2002). Estimación de Parámetros de Ecuaciones Diferenciales Estocásticas. Revista Mexicana de Economía y Finanzas, 1(1), pp. 83-91.

$\varnothing \mathrm{ksendal}, \mathrm{B} .(1998)$. Stochastic Differential Equations - An Introduction with Applications. 5th edition, Springer-Verlag, New York.

Shiryaev, A. N. (1999). Essential of Stochastic Finance: Facts, Models, Theory. World Scientific, River Edge, N. J.

Zane, O. (1994). Identification of Parameters with Convergence Rate for Bilinear Stochastic Differential Equations. Ulam Quarterly, 2(4), pp. 65-74. 